\title{
Correlaciones entre plasticidad, resistencias mecánicas, relaciones agua/cemento y proporciones de filler calizo en morteros de cemento
}

\section{Correlations among plasticity, mechanical strength, water/cement ratios and limestone filler content in cement mortars}

IGNACIO MENÉNDEZ y FERNANDO TRIVIÑO ICCET/CSIC

FRANCISCO HERNÁNDEZ ETSICCP. UPM.

ESPAÑA

Fecha de recepción: 24-IX-93

\section{$R E S U M E N$}

La adición del "filler" calizo al cemento portland, no sólo tiene influencia sobre las resistencias mecánicas de los morteros, sino que también actúa alterando los valores reológicos de los mismos.

Partiendo de un cemento portland al que se le añade "filler" calizo, en proporciones del 0 al $50 \%$, se han preparado morteros normalizados y se ha determinado su escurrimiento y fraguado (valores reológicos), estableciéndose la correlación entre éstos y el correspondiente contenido en "filler" calizo del cemento. Asimismo, sobre los morteros endurecidos se realizó un estudio por difracción de rayos $X$ y resistencias mecánicas. (1), (2), (9), (10), (11).
$S \cup M M A R Y$

Limestone filler addition to Portland cement does not only have an impact on the mechanical strength of mortars, but its action also alters their rheological values.

Taking a Portland cement as the basic material and incorporating different calcareous filler portions from 0 to $50 \%$, standard mortars were prepared, determining drainage and setting (rheological values) and establishing the correlations between these values and the respective limestone filler content. In addition, the hardened mortars were studied by $X$-ray diffraction and their mechanical strength was determined (1),(2), (9), (10), (11) .

\section{INTRODUCCIÓN}

Normalmente se ha trabajado con proporciones de "filler" calizo de hasta un $15 \%$. En la bibliografía se tienen datos abundantes de las resistencias mecánicas para proporciones del $15 \%$ y resultados aislados de los mismos para proporciones superiores, pero son muy escasas las referencias a ciertas propiedades reológicas de los morteros que se ven alteradas en función del porcentaje de "filler" calizo.

En nuestro trabajo se han estudiado las influencias de las adiciones de "filler" calizo al cemento portland sobre las resistencias

\section{INTRODUCTION}

Conventional filler content in these materials does not surpass $15 \%$. In the literature abundant data can be found referring to the mechanical strength of mortars with $15 \%$ filler content, but the data reporting on higher filler concentrations are scarce, as well as references to certain rheological properties of the mortars being altered as a consequence of calcareous filler incorporation.

In this research the effect of limestone filler addition to Portland cement on the mechanical strength of the resulting materials is studied, for 
mecánicas para proporciones del

0 al $50 \%$, en el se ha podido comprobar la tendencia a caídas de las resistencias mecánicas y se han contemplado otras alteraciones sufridas por los morteros frescos antes y durante su moldeo. También se hace mención a la necesidad de tener un conocimiento más amplio no sólo desde el punto de vista de las resistencias, sino de las propiedades reológicas tales como: plasticidad (escurrimientos), trabajabilidad, fraguado etc., así como fisuración, fenómenos que se producen como consecuencia de la adición de "filler" calizo en proporciones fundamentalmente superiores a las indicadas en el pliego RC-88 del (15\%). (3), (4), (5), (6), (7).

\section{PARTE EXPERIMENTAL}

\section{Preparación de las materias primas}

Se ha utilizado un cemento portland sin adiciones, obtenido a partir de un clínker molido y un yeso (sulfato cálcico dihidratado RA. Merck) mezclados en proporciones tales que la relación final de clínker/yeso fuera 9,34 (9,67\% en yeso), cantidad máxima tolerada según el pliego de cementos RC-88. Teniendo en cuenta que el contenido en $\mathrm{SO}_{3}$ del cemento portland era del $0,93 \%$ ( $2 \%$ en yeso), la adición de yeso fue del $7,67 \%$.

Una vez molido el clínker, se realizó el análisis granulométrico del mismo, y se observó que el $100 \%$ del tamaño de sus gránulos era inferior a $106 \mu \mathrm{m}$, y que el $93 \%$ lo era a $38 \mu \mathrm{m}$.

La caliza se molió separadamente y presentó el inconveniente de aglutinar sus partículas con lo que se falsea, en parte el tamaño de las mismas y en definitiva el tamaño de la muestra molida y por lo tanto su granulometría. Para evitarlo, se tamizaron los finos obtenidos repetidamente después de cada molienda pasándolos por el tamiz de $30 \mu \mathrm{m}$ en su totalidad, comprobando finalmente el tamaño máximo de la muestra.

El análisis químico del clínker dio los siguientes resultados:

Pérdida al fuego $=0,39 \%$;

$$
\begin{aligned}
& \mathrm{Fe}_{2} \mathrm{O}_{3}=3,45 \% ; \\
& \mathrm{SO}_{3}=0,93 \% ; \\
& \mathrm{SiO}_{2}=21,28 \% ; \\
& \mathrm{CaO}=65,86 \% ;
\end{aligned}
$$

filler portions from 0 to $50 \%$. The experimental data reveal the trend of mechanical strength to drop, as well as other alterations undergone by the fresh mortars prior to and during moulding. Furthermore the need of gaining deeper insight is highlighted, not only regarding mechanical strength, but moreover into other rheological properties, such as plasticity (drain rates), workability, hardening, cracking, etc,. i.e. into phenomena which arise as a consquence of calcareous filler incorporation in proportions substantially higher than those indicated in Standard Specification RC-88 (15\%), (3), (4), (5), (6), (7).

\section{EXPERIMENTAL}

\section{Preparation of raw materials}

A filler-free Portland cement was used, obtained from ground clinker and gypsum (dehydrated calcium sulphate supplied by RA. Merck), blended in such a way that the final clinker/gypsum ratio was 9.34 (9.67\% in gypsum), i.e. the maximum amount permitted according to Standards Specification RC-88. Taking into account that the S03 content of the Portland cement was $0.93 \%$ (2\% in gypsum) the actual gypsum addition amounted to $7.67 \%$.

Once the clinker had been ground, granulometric analysis revealed $100 \%$ grain size to be below $106 \mu \mathrm{m}, 93 \%$ of which was below $38 \mu \mathrm{m}$.

The limestone was ground separately and presented the inconvenient of particle agglutination, thus partially falsifying their grain size and definitely that of the ground sample and hence the granulometric data as a whole. In order to correct this defect, the fine particles obtained were screened several times through a $30 \mu \mathrm{m}$ mesh after each run through the grinder, until no residues were left, checking maximum sample size at the end of this process.

Chemical analysis composition of the clinker used yielded the following composition:

Firing loss $=0,39 \%$;

$$
\begin{aligned}
& \mathrm{Fe}_{2} \mathrm{O}_{3}=3,45 \% ; \\
& \mathrm{SO}_{3}=0,93 \% ; \\
& \mathrm{SiO}_{2}=21,28 \% ; \\
& \mathrm{CaO}=65,86 \% ;
\end{aligned}
$$


CaO libre = 1,21\%;

$\mathrm{Al}_{2} \mathrm{O}_{3}=5,59 \%$;

$\mathrm{MgO}=1,30 \%$

El clínker más el yeso, es decir el cemento así obtenido se mezcló posteriormente con el "filler" calizo en las proporciones del $0,5,10,15,20,30$, 40 y $50 \%$ de este último, formándose los respectivos cementos con adiciones.

Se obtuvieron los DRX del clínker, de la caliza, y de muestras de las series de probetas de mortero fabricadas, eliminando previamente la mayor parte posible de la arena mediante paso por el tamiz de $80 \mu \mathrm{m}$.

\section{PREPARACIÓN DE LOS MORTEROS}

Se fabricaron morteros normalizados con los ocho tipos de mezclas de cemento-"filler", indicadas anteriormente con arena normalizada UNE.

El mortero de cemento estaba constituido por $450 \mathrm{~g}$ de cemento, $1.350 \mathrm{~g}$ de arena normalizada UNE y $225 \mathrm{~g}$ de agua, con lo que se obtuvo una relación agua/cemento, en cada caso, de 0,50 .

En las condiciones citadas se ha determinado el escurrimiento de los morteros fabricados según norma UNE, obteniéndose en este caso una variación del mismo que se manifiesta claramente a partir de la proporción en "filler" añadido al cemento del $15 \%$ siempre para una relación agua/cemento constante igual a 0,50.

Una vez determinado el escurrimiento en las condiciones anteriormente citadas, se tomó como escurrimiento patrón el correspondiente al mortero de cemento con el $0 \%$ de "filler" calizo, y se variaron las relaciones agua/cemento de los restantes morteros con las distintas adiciones, a fin de mantener la constancia del escurrimiento tomado como referencia en los mismos.

Realizadas las anteriores medidas y sacadas las conclusiones de lo observado en el moldeo, se fabricaron probetas en ambos casos para determinar sus resistencias mecánicas a flexotracción y compresión a edades de 7, 28 y 90 días.

Las probetas fabricadas de dimensiones $4 \times 4 \times 16 \mathrm{~cm}$, se curaron en cámara húmeda a $21 \pm 2^{\circ} \mathrm{C}$ y $98 \%$ de $\mathrm{hr}$.
Free $\mathrm{CaO}=1,21 \%$;

$\mathrm{Al}_{2} \mathrm{O}_{3}=5,59 \%$;

$\mathrm{MgO}=1,30 \%$

The clinker-gypsum composite, i.e. the cement thus obtained, was then blended with the following calcareous filler portions: $0,5,10,15,20,30,40$ and $50 \%$, giving rise to the respective additive cement samples.

$X R D$ was applied to the pure clinker, the limestone and the blended mortar samples obtained, eliminating previously and as far as possible the sand residues by screening through an $80 \mu m$ mesh.

\section{MORTAR PREPARATION}

Standard mortars were prepared incorporating Spanish standard UNE sand into the eight types of cement-filler blends.

The cement mortar consisted of $450 \mathrm{~g}$ cement, $1,350 \mathrm{~g}$ standard UNE sand and $225 \mathrm{~g}$ water, thus achieving in each case a water/cement ratio of 0.50 .

Under the conditions described the drain rates were determined for the mortars prepared in conformity with the Spanish Standard UNE, obtaining rheological variations, which became clearly manifest as of $15 \%$ filler content at a constant water/cement ratio of 0.50 .

Having determined the drain rates as described above, the value corresponding to the zero calcareous filler mortar was chosen as the reference drain, varying the water/cement ratios of the other mortars containing different amounts of filler, in order to obtain a constant drain rate approaching the reference value in all the other samples.

Once the respective measurements had been recorded and conclusions had been arrived at in the light of the observations made during moulding, samples were prepared in both cases to the purpose of determining mechanical strength under bending stress and compression at ages 7 , 28 and 90 days.

The samples dimensioned $4 \times 4 \times 16 \mathrm{~cm}$ were cured in a humid chamber at $21 \pm 2^{\circ} \mathrm{C}$ and $98 \%$ r.h. 


\section{RESULTADOS DE LOS TRABAJOS EXPERIMENTALES}

Los valores de escurrimiento obtenidos en la mesa de sacudidas con los distintos cementos portland con adición descritos anteriormente y para una relación agua/cemento constante de 0,50 se dan en la tabla $I$.

En la tabla I se incluyen además las relaciones entre los escurrimientos de los morteros y la proporción de "filler" calizo en el cemento.

En la tabla II, se dan los valores para una relación agua/cemento obtenidos con los distintos tipos de cemento para un escurrimiento constante de $95 \pm 5 \%$, obtenido con el mortero sin adición de "filler" calizo, y dan además las dependencias entre la proporción de "filler" en el cemento y la relación agua/cemento obtenidas a escurrimiento constante.

Las probetas fabricadas, una vez curadas a 7, 28, 90 y 360 días, se sometieron a los ensayos mecánicos de flexotracción y compresión y sus resultados medios se encuentran en las tablas III y IV.

Los valores a flexotracción son las medias de los obtenidos en las roturas de tres probetas, y los de compresión son las medias de los valores obtenidos a partir de los seis trozos resultantes de las roturas a flexotracción.

\section{DRX}

Los trozos rotos de probetas resultantes de los ensayos mecánicos fueron triturados con martillo de plástico (teflón) y terminando con un tapón de goma, para impedir la rotura de las partículas, se tamizaron por un tamiz de $80 \mu \mathrm{m}$ separando el material cementante de la arena. Una vez preparada la muestra, se efectuaron los correspondientes diagramas de rayos $\mathrm{X}$. Los resultados expresados en cuentas/sg se encuentran en la tabla $\mathrm{V}$.

\section{DISCUSIÓN DE RESULTADOS}

\section{A) Escurrimientos a relación agua/cemento constante}

Para una relación agua/cemento constante e igual a 0,50 se produce una pérdida de trabajabilidad en el mortero, a medida que aumenta el porcentaje de "filler" calizo en el cemento, siendo dicha pérdida significativa a partir del $20 \%$. Para dicha cantidad hay que forzar manualmente la

\section{RESULTS FROM EXPERIMENTAL WORK}

The drain values obtained at shakeout for the different Portland cements with the filler additions described above and a constant water/cement ratio of 0.50 , are compiled in Table 1 .

In addition, Table I shows the correlations between the mortar drain values and filler content in the cement.

Table II lists the water/cement ratios obtained for the different cement types at a constant drain rate of $95 \pm 5 \%$, as obtained for the filler-free mortar. In addition, the dependency relationships are indicated existing betwen the filler portion in the cement and the water/cement ratios obtained for constant drain rate.

After ageing for 7, 28, 90 and 360 days the samples were subjected to mechanical strength tests (bending stress and compression), the mean results of which are recorded in Tables III and IV, respectively.

The bending stress data are the mean values obtained from the rupture of three samples; compression test results are indicated as the averages obtained from the six pieces (two for each sample) after bending to rupture.

\section{XRD}

Subsequent to these mechanical tests the broken samples were ground with a plastic hammer (teflon) and finishing with a rubber cap to avoid particle rupture. The ground material was then screened through an $80 \mu \mathrm{m}$ mesh separating the cement-like material from the sand. Subsequently the respective XRD diagrams were obtained from the samples prepared in this way. The results are expressed in counts/sec. and listed in Table V.

\section{DISCUSSION OF RESULTS}

\section{A) Drain rates at constant water/cement ratio}

For a constant water/cement ratio of 0.50 , the mortars suffer a workability loss, which increases proportionate to limestone filler portion in the cement, the loss becoming significant as of $20 \%$ filler content. As of this level mortar compaction has to be reinforced manually, especially for high 
TABLA I TABLE I

\begin{tabular}{|c|c|c|}
\hline $\begin{array}{l}\text { Relación (w/c) agua/cemento } \\
\text { (W/c ratio water/cement) }\end{array}$ & $\begin{array}{l}\text { Proporción de "filler" \% } \\
\text { (Filler content \%) }\end{array}$ & $\begin{array}{l}\text { Escurrimiento de los morteros \% } \\
\text { (Mortar drain rate \%) }\end{array}$ \\
\hline \multirow{8}{*}{$\begin{array}{c}0,50 \\
\text { constante } \\
\text { (constant) }\end{array}$} & 0 & $50 \pm 5$ \\
\hline & 5 & $42 \pm 5$ \\
\hline & 10 & $38 \pm 5$ \\
\hline & 15 & $32 \pm 5$ \\
\hline & 20 & $24 \pm 5$ \\
\hline & 30 & $8 \pm 5$ \\
\hline & 40 & $2 \pm 5$ \\
\hline & 50 & 0,0 \\
\hline
\end{tabular}

TABLA II TABLE ॥

\begin{tabular}{|c|c|c|}
\hline $\begin{array}{l}\text { Escurrimiento \% } \\
\text { (Drain rate \%) }\end{array}$ & $\begin{array}{l}\text { Porcentaje de "filler" } \\
\text { (Filler content \%) }\end{array}$ & $\begin{array}{l}\text { Relación agua/cemento w/c } \\
\text { (Water/cement ratio w/c) }\end{array}$ \\
\hline \multirow{8}{*}{$\begin{array}{l}95 \pm 5 \% \\
\text { constante } \\
\text { (constant) }\end{array}$} & 0 & 0,52 \\
\hline & 5 & 0,53 \\
\hline & 10 & 0,56 \\
\hline & 15 & 0,59 \\
\hline & 20 & 0,60 \\
\hline & 30 & 0,61 \\
\hline & 40 & 0,67 \\
\hline & 50 & 0,70 \\
\hline
\end{tabular}


TABLA III TABLE III

Valores experimentales medios de las resistencias mecánicas

(Mean experimental mechanical strength values)

\begin{tabular}{|c|c|c|c|c|c|c|c|c|c|}
\hline \multicolumn{10}{|c|}{$w / c=0,50$} \\
\hline \multicolumn{2}{|c|}{ Cemento (Cement) } & \multicolumn{4}{|c|}{$\begin{array}{c}\text { R. Flexotracción } \\
\text { (Bending stress) } \\
\mathrm{N} / \mathrm{mm}^{2}\end{array}$} & \multicolumn{4}{|c|}{$\begin{array}{c}\text { R. Compresión } \\
\text { (Compression) } \\
\mathrm{N} / \mathrm{mm}^{2}\end{array}$} \\
\hline \multirow{2}{*}{$\begin{array}{c}\text { Clínker + yeso } \\
\text { (Clinker + gypsum) }\end{array}$} & \multirow{2}{*}{$\begin{array}{c}\text { "Filler" calizo } \\
\text { (Calcareous "filler") } \\
\%\end{array}$} & \multicolumn{4}{|c|}{$\begin{array}{c}\text { Edad días } \\
\text { (Ageing/days) }\end{array}$} & \multicolumn{4}{|c|}{$\begin{array}{c}\text { Edad días } \\
\text { (Ageing/days) }\end{array}$} \\
\hline & & 7 & 28 & 90 & 360 & 7 & 28 & 90 & 360 \\
\hline 100 & 0 & 6,6 & 7,5 & 8,1 & 8,3 & 42,3 & 58,6 & 68,4 & 71,6 \\
\hline 95 & 5 & 6,0 & 6,9 & 7,6 & 7,9 & 37,0 & 53,7 & 62,0 & 68,0 \\
\hline 90 & 10 & 5,4 & 6,6 & 7,4 & 7,5 & 34,5 & 49,4 & 57,4 & 64,4 \\
\hline 85 & 15 & 5,1 & 6,4 & 7,2 & 7,1 & 33,0 & 46,9 & 54,7 & 60,9 \\
\hline 80 & 20 & 4,9 & 6,0 & 7,0 & 6,6 & 29,0 & 43,3 & 50,0 & 57,3 \\
\hline 70 & 30 & 4,6 & 5,6 & 6,2 & 5,8 & 25,1 & 37,5 & 41,5 & 50,1 \\
\hline 60 & 40 & 3,9 & 4,5 & 5,5 & 5,0 & 20,7 & 31,5 & 34,0 & 43,0 \\
\hline 50 & 50 & 3,0 & 3,1 & 4,8 & 4,2 & 16,0 & 23,8 & 26,0 & 35,8 \\
\hline
\end{tabular}

compactación del mortero, sobre todo cuando las proporciones de "filler" son del 30 al $50 \%$, pues deja de ser operativo el proceso de compactación descrito en la RC-88.

Las resistencias mecánicas se hacen claramente menores a partir de un contenido de "filler" calizo en el cemento del $20 \%$. (3), (7).

\section{B) Variación de la relación agua/cemento a escurrimiento constante}

Si queremos que se mantenga la trabajabilidad de los morteros al variar los porcentajes de "filler" calizo en el cemento, es necesario que la relación agua/cemento varie a medida que aumenta el contenido de "filler" calizo. Las consecuencias observadas son las previstas: aumento de la relación agua/cemento $y$, por tal motivo nueva pérdida de las resistencias mecánicas que sufren los morteros. Lo descrito nos permite hacer la siguiente observación indicada ya en la filler content between 30 and $50 \%$, as of which the compaction process described in Specification $R C-88$ is no longer operative.

Mechanical strength clearly diminishes as of $20 \%$ calcareous filler content (3), (7).

\section{B) Variation of the water/cement ratio at constant drain rate}

If constancy of the workability value is to be achieved in the mortars at variable filler content, the water/cement ratio will change as a function of filler content. The behaviour observed experimentally confirms this theoretical expectation. The water/cement rate increases with filler content and hence motivates further loss in mechanical strength in the mortars. These observations legitimate the following statement in agreement with the literature (11), (12). For 
TABLA IV TABLE IV

Valores experimentales medios de las resistencias mecánicas

(Mean experimental mechanical strength values)

\begin{tabular}{|c|c|c|c|c|c|c|c|c|c|}
\hline \multicolumn{10}{|c|}{$w / c=0,52$} \\
\hline \multicolumn{2}{|c|}{ Cemento (Cement) } & \multicolumn{4}{|c|}{$\begin{array}{c}\text { R. Flexotracción } \\
\text { (Bending stress) } \\
\mathrm{N} / \mathrm{mm}^{2}\end{array}$} & \multicolumn{4}{|c|}{$\begin{array}{c}\text { R. Compresión } \\
\text { (Compression) } \\
\mathrm{N} / \mathrm{mm}^{2}\end{array}$} \\
\hline \multirow{2}{*}{$\begin{array}{c}\text { Clínker + yeso } \\
\text { (Clinker + gypsum) }\end{array}$} & \multirow{2}{*}{$\begin{array}{c}\text { "Filler" calizo } \\
\text { (Calcareous "filler") } \\
\%\end{array}$} & \multicolumn{4}{|c|}{$\begin{array}{c}\text { Edad días } \\
\text { (Ageing/days) }\end{array}$} & \multicolumn{4}{|c|}{$\begin{array}{c}\text { Edad días } \\
\text { (Ageing/days) }\end{array}$} \\
\hline & & 7 & 28 & 90 & 360 & 7 & 28 & 90 & 360 \\
\hline 100 & 0 & 6,1 & 7,2 & 7,4 & 7,6 & 40,9 & 55,4 & 56.5 & 61,6 \\
\hline \multicolumn{10}{|c|}{$w / c=0,53$} \\
\hline 95 & 5 & 5,7 & 6,4 & 6,0 & 7,2 & 35,6 & 49,5 & 56,6 & 60,7 \\
\hline \multicolumn{10}{|c|}{$w / c=0,56$} \\
\hline 90 & 10 & 5,3 & 6,0 & 6,3 & 6,6 & 32,1 & 43,5 & 45,4 & 48,7 \\
\hline \multicolumn{10}{|c|}{$w / c=0,59$} \\
\hline 85 & 15 & 4,7 & 5,9 & 6,1 & 6,4 & 27,2 & 36,8 & 39,1 & 41,9 \\
\hline \multicolumn{10}{|c|}{$w / c=0,60$} \\
\hline 80 & 20 & 4,3 & 5,5 & 5,6 & 6,1 & 22,0 & 29,7 & 35,3 & 37,9 \\
\hline \multicolumn{10}{|c|}{$w / c=0,61$} \\
\hline 70 & 30 & 3,9 & 4,5 & 5,0 & 5,3 & 20,0 & 27,0 & 28,8 & 30,3 \\
\hline \multicolumn{10}{|c|}{$w / c=0,67$} \\
\hline 60 & 40 & 2,5 & 3,3 & 3,4 & 3,7 & 12,0 & 16,2 & 18,1 & 19,1 \\
\hline \multicolumn{10}{|c|}{$w / c=0,70$} \\
\hline 50 & 50 & 2,0 & 2,3 & 2,6 & 2,9 & 8,8 & 11,9 & 12,6 & 13,3 \\
\hline
\end{tabular}


TABLA V TABLE V

\begin{tabular}{|c|c|c|c|c|c|c|}
\hline \multicolumn{2}{|c|}{ Cuentas $2 \theta$ (Counts 2 $\theta$ ) } & \multirow{2}{*}{$\begin{array}{r}29,40 \\
\mathrm{CaCO}_{3}\end{array}$} & \multirow{2}{*}{$\begin{array}{c}34,00 \\
\mathrm{Ca}(\mathrm{OH})_{2}\end{array}$} & \multirow{2}{*}{$\begin{array}{c}\quad 9,11 \\
\begin{array}{c}\text { Ettringita } \\
\text { (Ettringite) }\end{array}\end{array}$} & \multirow{2}{*}{$\begin{array}{l}51,0 \\
\mathrm{C}_{3} \mathrm{~S}\end{array}$} & \multirow{2}{*}{$\begin{array}{r}11,70 \\
\mathrm{Ca}\left(\mathrm{SO}_{4}\right.\end{array}$} \\
\hline $\begin{array}{c}\text { "Filler" } \\
\text { calizo } \\
\text { (Calcareous } \\
\text { "filler") }\end{array}$ & $\begin{array}{l}\text { Edad días } \\
\text { (Age days) }\end{array}$ & & & & & \\
\hline \multirow{4}{*}{0} & 7 & 105 & 580 & 77 & 14 & - \\
\hline & 28 & 210 & 529 & 58 & 62 & - \\
\hline & 90 & 133 & 476 & 64 & - & - \\
\hline & 360 & 140 & 502 & 63 & - & - \\
\hline \multirow{4}{*}{5} & 7 & 173 & 557 & 82 & 48 & - \\
\hline & 28 & 317 & 520 & 72 & - & - \\
\hline & 90 & 170 & 542 & 63 & - & 46 \\
\hline & 360 & 190 & 534 & 77 & - & 50 \\
\hline \multirow{4}{*}{10} & 7 & 200 & 548 & 92 & 36 & 28 \\
\hline & 28 & 317 & 498 & 84 & - & 41 \\
\hline & 90 & 202 & 539 & 67 & - & 67 \\
\hline & 360 & 214 & 540 & 69 & - & 72 \\
\hline \multirow{4}{*}{15} & 7 & 271 & 531 & 58 & 26 & 48 \\
\hline & 28 & 270 & 632 & 78 & - & 63 \\
\hline & 90 & 293 & 526 & 87 & - & 84 \\
\hline & 360 & 272 & 541 & 76 & - & 86 \\
\hline \multirow{4}{*}{20} & 7 & 320 & 554 & 89 & 32 & 53 \\
\hline & 28 & 496 & 549 & 89 & - & 80 \\
\hline & 90 & 320 & 580 & 83 & - & 80 \\
\hline & 360 & 356 & 563 & 82 & - & 82 \\
\hline
\end{tabular}


TABLA V TABLE V (Continuación)

\begin{tabular}{|c|c|c|c|c|c|c|}
\hline \multicolumn{2}{|c|}{ Cuentas $2 \theta$ (Counts $2 \theta$ ) } & \multirow{2}{*}{$\begin{array}{l}29,40 \\
\mathrm{CaCO}_{3}\end{array}$} & \multirow{2}{*}{$\begin{array}{c}34,00 \\
\mathrm{Ca}(\mathrm{OH})_{2}\end{array}$} & \multirow{2}{*}{$\begin{array}{c}9,11 \\
\\
\text { Ettringita } \\
\text { (Ettringite) }\end{array}$} & \multirow{2}{*}{$\begin{array}{l}51,0 \\
c_{3} S\end{array}$} & \multirow{2}{*}{$\begin{array}{c}11,70 \\
\mathrm{Ca}\left(\mathrm{SO}_{4}\right)\end{array}$} \\
\hline $\begin{array}{c}\text { "Filler" } \\
\text { calizo } \\
\text { (Calcareous } \\
\text { "filler") }\end{array}$ & $\begin{array}{l}\text { Edad días } \\
\text { (Age days) }\end{array}$ & & & & & \\
\hline \multirow{4}{*}{30} & 7 & 403 & 545 & 63 & - & 40 \\
\hline & 28 & 507 & 673 & 102 & - & 90 \\
\hline & 90 & 383 & 559 & 72 & - & 107 \\
\hline & 360 & 406 & 540 & 67 & - & 103 \\
\hline \multirow{4}{*}{40} & 7 & 548 & 609 & 67 & - & 50 \\
\hline & 28 & 612 & 703 & 77 & - & 89 \\
\hline & 90 & 560 & 541 & 82 & - & 172 \\
\hline & 360 & 592 & 562 & 76 & - & 96 \\
\hline \multirow{4}{*}{50} & 7 & 673 & 464 & 56 & - & 48 \\
\hline & 28 & 752 & 718 & 70 & - & 96 \\
\hline & 90 & 710 & 600 & 118 & - & 170 \\
\hline & 360 & 690 & 549 & 58 & - & 132 \\
\hline
\end{tabular}

bibliografía (11) (12). Para ciertos porcentajes de "filler" calizo podemos considerar que obtenemos un nuevo cemento que, cumpliendo el pliego, tiene resistencias mecánicas inferiores al cemento de partida o, lo que es lo mismo, a las obtenidas con el cemento portland sin adiciones o con adiciones en porcentajes inferiores.

\section{C) Resistencias mecánicas}

\section{1c) A relación agua/cemento constante}

Relación agua/cemento $=0,50$

Se producen pérdidas en las resistencias mecánicas a compresión a la edad de 28 días del orden del $50 \%$ entre el mortero con cemento patrón y el mortero con el 50 \% de "filler" calizo en el cemento. certain calcareous filler percentages a new cement is obtained which, when complying with the Standard Specification, possesses a lower mechanical strength than the input cement, in other words lower than those of the additive-free Portland cement or when filled at lower percentages.

\section{C) Mechanical strength}

1c) A constant water/cement ratio

Water/cement ratio $=0.50$

The compression test at age 28 days revealed a mechanical strength loss in the order of $50 \%$ for the sample with $50 \%$ filler content, as compared to the reference mortar. 
La trabajabilidad del mortero se hace cada vez más difícil, llegando a ser imposible la compactación de los morteros mediante el método descrito en el RC 88 para porcentajes de "filler" del orden del 40 y $50 \%$.

Es decir, la pérdida de resistencia está relacionada con el incremento del porcentaje de "filler" calizo y con la pérdida de trabajabilidad a igual relación agua/cemento.

\section{2c) A escurrimiento constante}

Escurrimiento constante igual a $95 \pm 5 \%$

Variación de la relación agua cemento aumentando con el porcentaje de "filler" calizo pasando de 0,52 para el $0 \%$ de "filler" a 0,70 para el $50 \%$ de "filler". Las pérdidas de resistencia a compresión en este caso son notables. Sabemos, por el apartado anterior, que a medida que aumenta el porcentaje de "filler" decrecen las resistencias, alcanzando pérdidas a 28 días del orden del $50 \%$ para porcentajes de "filler" del $50 \%$, pero en este caso dichas pérdidas se hacen notablemente más significativas al aumentar la relación agua/cemento, con el fin de mantener el mismo escurrimiento en la mesa de sacudidas.

En el caso de que las pérdida de resistencia al aumentar el "filler" y mantener el escurrimiento de los morteros dentro de unos límites, estén dentro de los valores que cumplen con el pliego, podemos considerar que dichos cementos son distintos a los anteriores por poseer resistencias mecánicas distintas (en este caso inferiores), pero son utilizables al cumplir los requisitos especificados.

\section{D) D.R.X}

Si no consideramos la variable sílice que presentan las muestras, y utilizando los resultados de la tabla $\mathrm{V}$-los correspondientes al $\mathrm{CaCO}_{3}, \mathrm{Ca}(\mathrm{OH})_{2}$, ettringita, $\mathrm{C}_{3} \mathrm{~S}\left(3 \mathrm{CaOSiO}_{2}\right)$ y yeso-, se observa que al aumentar la relación $\mathrm{CaCO}_{3}$ /clínker + yeso, los resultados indican que hay una correlación entre los valores obtenidos por DRX y las cantidades añadidas de $\mathrm{CaCO}_{3}$. (1), (2).

\section{E) Observaciones}

La resistencia pasa de $58,6 \mathrm{~N} / \mathrm{mm}^{2}$ para el $0 \%$ de "filler" calizo, a 29,3 N/mm², para el $50 \%$ de "filler", a relación $w / c$ constante $=0,5$.
Workability becomes more and more difficult to the point of making mortar compaction unfeasible following the method described in $\mathrm{RC}-88$, for filler portions between 40 and $50 \%$.

This means that the loss in strength correlates to limestone filler increments and the respective loss in workability, the water/cement rate remaining constant.

2c) A constant drain rate

Constant drain rate $=95 \pm 5 \%$

The water/cement ratio, which increases with growing filler content, shows a variation from 0.52 for zero filler content to 0.70 for $50 \%$ filler portion. The loss in compression strength in this latter case is remarkable. In the preceding paragraph mechanical strength was described as inversely proportionate to filler content, strength losses in the order of $50 \%$ being recorded for the $50 \%$ filler sample aged 28 days. These strength losses become, however, notably more significant when they occur in combination with an increase in the water/cement ratio, in order to maintain the drain rate constant at shakeout.

Considering the case where the strength losses due to higher filler content remain within the tolerances laid down in the Specification and the drain rate is also maintained within certain limits, it is safe to consider these cements as different from the reference materials, owing to the fact that their mechanical strength values are different (in our case they are lower than the reference values), yet can still be employed, as they remain within specs.

\section{D) XRD}

Discarding the silica variable in the samples and utilizing the data contained in Table $\mathrm{V}$ -corresponding to $\mathrm{CaCO}_{3}, \mathrm{Ca}(\mathrm{OH})_{2}$, ettringite, $\mathrm{C}_{3} \mathrm{~S}\left(3 \mathrm{CaOSiO}_{2}\right)$ and gypsum- it can be observed that, when increasing the $\mathrm{CaCO}_{3}$ /clinker + gypsum ratio, there is a good correlation between the $X R D$ values and the amounts of $\mathrm{CaCO} 3$ added (1), (2).

\section{E) Observations}

Strength values decrease from $58.6 \mathrm{~N} / \mathrm{mm}^{2}$ for zero filler portion to $29.3 \mathrm{~N} / \mathrm{mm}^{2}$ for the $50 \%$ filler sample, at constant $w / c$ ratio $=0.5$. 
La pérdida de resistencia entre el mortero con cemento patrón y el mortero con un $50 \%$ de "filler" calizo en el cemento, es del orden del $50 \%$.

El escurrimiento del mortero pasa del $50 \pm 5 \%$ para el $0 \%$ de "filler" calizo en el cemento, al $0 \%$ para un $50 \%$ de "filler" calizo en el cemento.

La relación w/c pasa de 0,52 para el $0 \%$ de "filler" calizo, a 0,70 para el $50 \%$ en "filler" a escurrimiento constante, y se produce una pérdida de resistencia a la compresión del orden del $78,5 \%$, a la edad de 28 días, entre el mortero con cemento patrón y el mortero con el $50 \%$ de "filler" calizo, es decir, a escurrimiento constante las resistencias a compresión decrecen en un $29 \%$ más sobre el $50 \%$ que se obtiene al añadir solamente "filler" a relación $\mathrm{w} / \mathrm{c}=$ constante.

La variación de la resistencia a 28 días en función del porcentaje de "filler" calizo manteniendo fija la relación agua/cemento o variando la misma para conseguir igual escurrimiento se puede ver en la Fig. 1.
The strength loss gradient between the mortar based on the reference cement and the $50 \%$ filler mortar amounts to $50 \%$.

The drain rate of the mortars decreases from $50 \pm 5 \%$ for the unfilled sample to $0 \%$ for the $50 \%$ lime-filled mortar.

The $w / c$ ratio rises from 0.52 for zero filler content to 0.70 for the $50 \%$ filler composite at constant drain rate, giving rise to a compression strength loss of $78.5 \%$ at 28 days, i.e. at constant drain rate compression strength decreases by $29 \%$ on top of the $50 \%$ loss due to simple filler addition at constant $w / c$ ratio.

Strength variation at 28 days as a function of filler content at constant water/cement ratio, or varying the latter to achieve a constant drain rate is shown in Fig. 1:

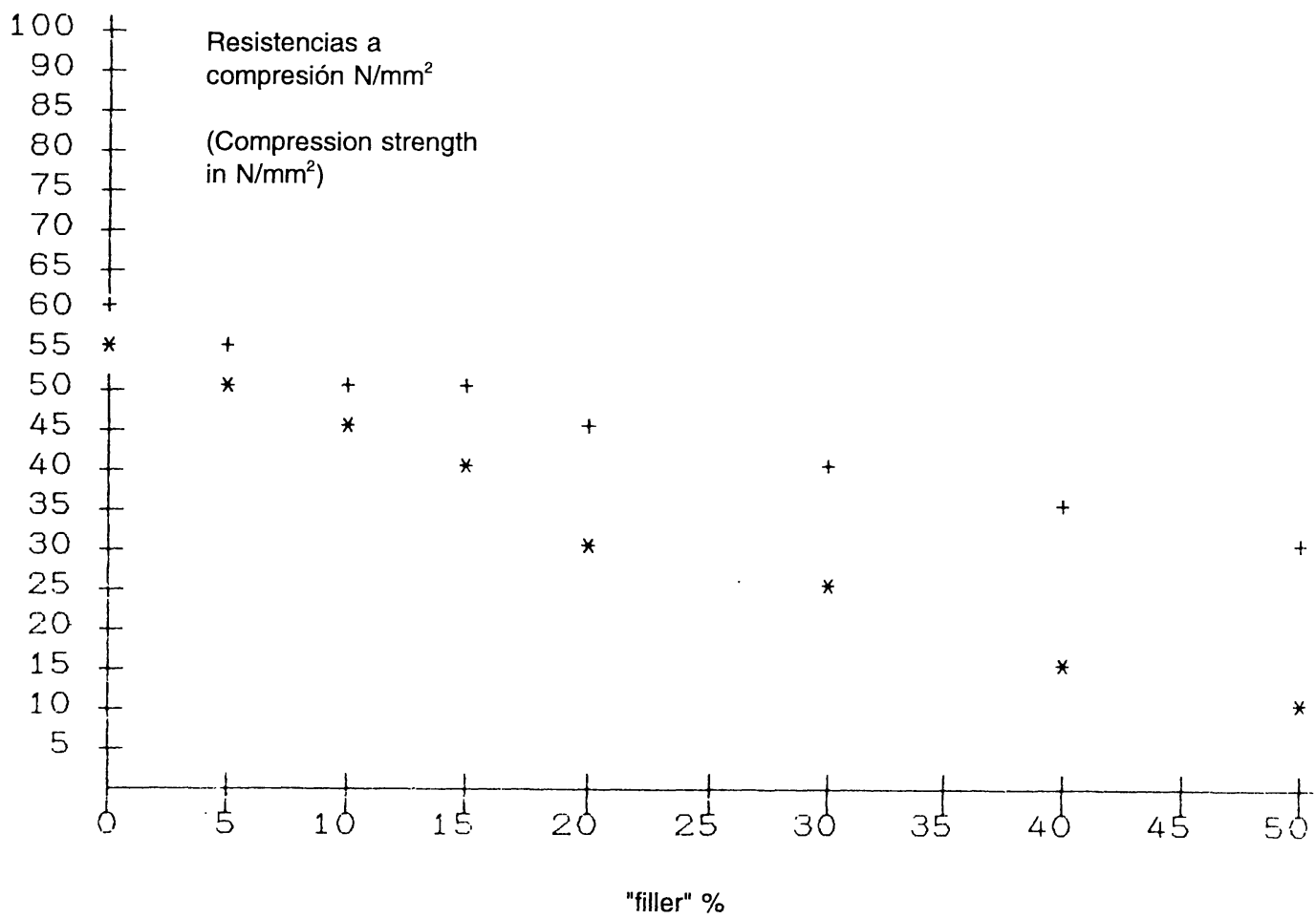

+ Resistencias a compresión a la edad de 28 días manteniendo la relación agua/cemento constante. (Compression strength at age 28 days maintaining the water/cement ratio constant).

* Resistencias a compresión a la edad de 28 días manteniendo el escurrimiento en la mesa de sacudidas constante. (Compression strength at age 28 days maintaining the drain rate at shakeout constant).

Fig. 1 


\section{CONCLUSIONES}

A) De los resultados obtenidos experimentalmente, deducimos que:

1. $\left.{ }^{\circ}\right)$ La trabajabilidad de los morteros se hace cada vez más difícil a medida que aumenta el porcentaje de "filler" calizo.

2. ${ }^{\circ}$ Para valores superiores al $20 \%$ en "filler" calizo, el método de compactación descrito en el RC-88 para una relaciones agua/cemento de 0,5 es insuficiente.

$3 .^{\circ}$ ) Los escurrimientos en la mesa de sacudidas para la misma relación agua/cemento, varían desde el $50 \pm 5$ para el $0 \%$ de contenido en "filler" calizo, al $0 \%$ de escurrimiento para el $50 \%$ de "filler" calizo.

4. $\left.{ }^{\circ}\right)$ Si mantenemos iguales los escurrimientos para distintas proporciones de "filler" calizo, las relaciones agua/cemento varían desde 0,52 hasta 0,70 , obteniéndose grandes descensos de resistencia a igual escurrimiento (trabajabilidad).

5. ${ }^{\circ}$ Las resistencias mecánicas no varían significativamente para contenidos de "filler" calizo inferiores al $20 \%$, pero sí varía su escurrimiento (trabajabilidad).

Las caídas de resistencia se acentúan con la edad para proporciones de "filler" calizo superiores al $15 \%$.

$\left.6 .{ }^{\circ}\right)$ Las elevadas resistencias de un cemento $\sin$ adición pueden disminuirse con adiciones de "filler" calizo, de forma que se puede obtener otro cemento que cumpla el pliego con resistencias mecánicas menores.

7..$^{\circ}$ La demanda de agua aumenta al aumentar el contenido en "filler" calizo.

\section{B) Del estudio de DRX, se deduce que:}

$\left.1 .{ }^{\circ}\right)$ No ha tenido lugar la formación de carboaluminatos en ninguna de las muestras. Muy posiblemente debido a que el yeso añadido no permite dicha formación favoreciendo la de ettringita.

2. ${ }^{\circ}$ El contenido en hidróxido cálcico no se altera con el tiempo de curado.

3. ${ }^{\circ}$ El yeso detectado aumenta para las edades de 90 días cuando los porcentajes de "filler" calizo son del 30,40 ó del $50 \%$, lo cual

\section{CONCLUSIONS}

A) In the light of the experimental data it is legitimate to conclude:

1. $\left.{ }^{\circ}\right)$ Mortar workability becomes increasingly difficult as a function of calcareous filler increments.

2. ${ }^{\circ}$ For filler portions above $20 \%$ the compaction method described in Standard Specification $R C-88$ for w/c ratios of 0.5 proves to be insufficient.

3. ${ }^{\circ}$ Drain rates at shakeout for equal $w / c$ ratio varies from $50 \pm 5$ for the filler-free sample to zero drain for the $50 \%$ filler sample.

4..$\left.^{\circ}\right)$ Maintaining the drain rate constant and varying calcareous filler content, the $\mathrm{w} / \mathrm{c}$ ratio varies between 0.52 and 0.70 causing the strength values to decrease remarkably at constant drain (workability).

5. $\left.{ }^{\circ}\right)$ Mechanical strength does not vary significantly for filler content below $20 \%$; drain rate, however, does vary.

The drops in strength become more prominent with ageing for filler portions above $15 \%$.

6. ${ }^{\circ}$ The high strength of additive-free cement can be reduced by means of limestone filler addition, in such a way that a new cement is obtained which still complies with the specifications, yet with lower mechanical strength values.

7.) Water demand increases proportionate to filler content.

B) The XRD study revealed:

$\left.1 .^{\circ}\right)$ Carboaluminate formation was not detected in any of the samples. This is possibly due to the fact that the gypsum added inhibits this formation favouring ettringite instead.

2. ${ }^{\circ}$ The calcium hydroxide content is not altered by ageing.

3. $\left.{ }^{\circ}\right)$ The gypsum detected increases as of age 90 days, when filler content is 30,40 or $50 \%$, which is indicative of ettringite 
indica la descomposición de la ettringita, que por otro lado tiende a disminuir.

\section{AGRADECIMIENTO}

Los autores agradecen a la Dirección General de Investigación Científica y Técnica el apoyo económico prestado para la elaboración de este proyecto PB 89-0189, sin el cual no se podría haber realizado. decomposition, which, in addition, tends to diminish.

\section{ACKNOWLEDGEMENTS}

The authors gratefully acknowledge financial support of this project (PB 89-0189) by the Directorate General of Scientific and Technological Research (Dirección General de Investigación Cientifica y Técnica), without which this research would not have materialized.

\section{BIBLIOGRAFÍA}

[1] BOBROSKI: "Limestone substitutes for gypsum as a cement ingredient" 6.5. Rock Products, pp. 64/67. 1967.

[2] T. GACESA y otros: "Calcaire comme addition and cement". Cement 21 (3-4) pp. 107-113. 1979.

[3] M. ${ }^{a}$ J. ESCORIHUELA; F. TRIVIÑO; I. MENÉNDEZ; F. HERNÁNDEZ; N. HURTADO; M. ${ }^{a}$ A. MARTÍN: "The efect of calcareous filler on portland cement mortars". Vol 43. n.․ 229, enero/febrero/marzo 1993. Materiales de Construcción, ICCET/CSIC, pp. 199/205.

[4] EIVING HOGNESTAD: "Calcite as concrete and mixture". Journal of the American Concrete Institute. Vol 25. n. 9 , pp. 801/803. 1954.

[5] CDTI-OFICEMEN: "Conclusiones sobre Cementos de adición", Nov. 1980.

[6] N. SETTER and I. SOROKA: "The effect of fillers on strength of Cement Mortars". Cement and Concrete Research, Vol 7, pp. 449/456, 1977.

[7] I. SOROKA and N. STERN: "Calcareons filler and the compressive strength of Portland Cement". Cement and Concrete Research. Vol 6, pp. 367/376. 1976.

[8] "El punto sobre las nuevas normas de cemento". Ciments et Chaux n.ำ 697. 1980.

[9] P. P. BUDNIKOV, V. M. KOLBASOV: "Hydration of the Aluminous Minerals of Portland Cement in the presence of finely ground carbonates". Cemen and Lime Manufature, pp. 60/61, julio 1962.

[10] LUISSINO CASSINO, GIUSEPPE PINTOR: "Indagine sul differente comportamento degli inerti silicico e calcareo". II Cemento, pp. 255/262, abril 1972.

[11] D. INGRANM and KENNETH E. DAUGHERTY: A review of limestons additions to portland cement and concrete. Cement and Concrete Research 143, pp. 165/170, 1991.

[12] J. BOMBLED: "Influence des fillers sur les propietes des mortiers et des betons". Ciment, Beton, Plâtre, Chaux, n. 738 , pp. $5 / 82$.

[13] I. MENÉNDEZ, F. HERNÁNDEZ, F. TRIVIÑO: "Influencia del "filler" calizo sobre el fraguado del cemento portland". Vol. 43, n.ำ 231, julio/agosto/septiembre 1993. Materiales de Construcción. ICCET/CSIC.

[14] F. TRIVIÑO, F. HERNÁNDEZ, I. MENÉNDEZ: "Influencia del "filler" calizo en las propiedades de los morteros a resistencia constante. Vol 44, n.․․ 232 octubre/noviembre/diciembre 1993. Materiales de Construccion. ICCET/CSIC. 